\title{
La Noción de Evaluación Eudaimonista en la Teoría Cognitiva de las Emociones de Martha Nussbaum ${ }^{1}$
}

\author{
Marta Gil
}

\begin{abstract}
RESUMEN: En este artículo se presenta la evaluación eudaimonista como una articulación entre el agente y los objetos del mundo. Sostendremos que, gracias a esta noción, la teoría de las emociones de Nussbaum es adecuada para dar cuenta de la función adaptativa de las emociones, por lo que las teorías no cognitivas no resultarían superiores en este aspecto. Esta noción serviría, asimismo, para no dejar de lado los aspectos cognitivos de la emoción. Veremos cómo las ideas de Nussbaum se basan en las de las teorías evaluativas provenientes de la psicología, para después desplegar su propuesta en detalle. En la exposición, también trataremos otras cuestiones, entre ellas, la racionalidad de las emociones, la relación entre éstas y el florecimiento humano, o la vinculación de las mismas con nuestra condición vulnerable.
\end{abstract}

PALABRAS CLAVE: Emociones. Martha Nussbaum. Teorías cognitivas. Evaluación. Eudaimonía. Racionalidad.

\section{UNA BREVE DIGRESIÓN SOBRE LAS TEORÍAS DE LA EMOCIÓN}

A pesar de que existen tantas teorías de la emoción como autores se han dedicado a escribir sobre las mismas, en la actualidad es habitual distinguir simplemente entre teorías cognitivas y teorias no cognitivas de la emoción. Las emociones tienen una naturaleza dual: por un lado, son meros impulsos, automatismos que compartimos con el resto de criaturas de reino animal y que están al servicio de la supervivencia, pero, por otro, las emociones son un entramado complejo, que no sólo entraña cambios corporales y una función adaptativa, sino también aquellas sutilezas propias de la inteligencia humana (recuerdos, imaginación, pensamientos, etc.). Así, un primer conjunto de teóricos describe las emociones en función del papel que cumplen en nuestra supervivencia, pero no suelen estar interesados en los aspectos normativos relacionados con las mismas (por ejemplo: ¿qué emociones merece la pena cultivar y por qué?, ¿̨existen emociones sospechosas de socavar los principios de

\footnotetext{
${ }^{1}$ El presente trabajo ha sido realizado en el marco del proyecto de la Universidad de Valencia FFI201021639-C02-01 Ética del discurso, política democrática y neuroética (Investigadora Principal: Adela Cortina Orts), financiado por el Ministerio de Economía y Competitividad.

http://dx.doi.org/10.1590/S0101-317320160003000011

${ }^{2}$ Departamento de Filosofía del Derecho, Moral y Política. Universidad de Valencia. Avda. Blasco Ibáńez, 30, 6ª planta. 46010 Valencia, España. E-mail: marta.gil@uv.es
} 
respeto mutuo y convivencia entre seres humanos?). Estos teóricos se centran, habitualmente, en aquello que ha dado en denominarse emociones básicas. ${ }^{3}$ Estas emociones serían la ira, la repugnancia, el miedo, la alegría, la tristeza, y la sorpresa. Dado que el resto de emociones no es susceptible de ser interpretado conforme a este esquema explicativo por incorporar elementos más complejos (por ejemplo, ¿cómo dar cuenta de la vergüenza si pretendemos ceñirnos exclusivamente a su función adaptativa? ¿no será preciso remitirse también a ideas tales como la imagen que uno tiene de sí mismo o la necesidad de ser reconocido y respetado por nuestros pares?), dicho esquema resulta alicorto si de lo que se trata es de comprender las complejidades de la emoción humana. ${ }^{4}$

En lo que respecta a las teorías cognitivas, éstas darían cuenta de las emociones en términos de las creencias o pensamientos con los que éstas se identifican o relacionan. Uno de los puntos débiles de estas teorías es que no acaba de quedar claro qué es exactamente lo que debemos entender por “cogniciones”(¿proposiciones lingüísticas?, ¿juicios con forma no lingüística?, ¿algún tipo de transmisión de información?). Sin embargo, la concepción de las emociones que presentan las teorías cognitivas es rica y compleja, y tiene en cuenta la proyección de ideas y valores, así como otros aspectos de la vida mental, como los recuerdos o las creencias, que se relacionan con la emoción de formas diversas. La teoría de las emociones de Martha Nussbaum se situaría en este último grupo.

No obstante, hay que tener presente que la dicotomía entre teorías cognitivas y teorías no cognitivas de la emoción no es tan radical, ya que los autores suelen incorporar elementos muy diversos a sus teorías de la emoción. Por ejemplo, Paul Ekman, cuya concepción de las emociones básicas o affect programs se encuentra en la base de las teorías no cognitivas de la emoción, defiende una teoría muy equilibrada. El propio autor denomina a su explicación de las emociones "neurocultural", haciendo hincapié en la doble vertiente, biológica y social, de las mismas (EKMAN, 1980, p. 80). Para Ekman, la respuesta emocional es algo "[...] breve, y, con frecuencia, rápido, organizado, complejo y difícil de controlar”. Estas respuestas se darían de forma automática a gran velocidad, y los affect programs serían los encargados de coordinar este conjunto de respuestas complejas, siendo el mecanismo que almacena patrones y dirige las respuestas cuando aparecen. Las emociones son complejas porque involucran

\footnotetext{
${ }^{3}$ Este conjunto de emociones fue propuesto por Paul Ekman, a quien varios de estos autores suelen tomar como referente. Ver Ekman (1999).

${ }^{4}$ Para saber más acerca de la perspectiva no cognitiva, el lector puede dirigirse a: Plutchik (1980), Zajonc (1980; 1984), Griffiths (1997); Delancey (2002), Goldie (2002); Prinz (2003, 2004a, 2004b, 2007).
} 
respuestas músculoesqueléticas, faciales, vocales, etc., pero también otro tipo de fenómenos como lo recuerdos, las imágenes, las expectativas o la valoración de las circunstancias. Ekman (1980, p. 84) señala que, “[...] en ocasiones, la evaluación de lo que está ocurriendo es lenta, deliberada y consciente”, y que, en consecuencia, "[...] la cognición juega un papel importante en la determinación de lo que ocurra." (EKMAN, 1980, p. 84).

De forma similar, Lazarus y Nussbaum son teóricos cognitivistas, pero no por ello dejan de lado la función adaptativa y los aspectos biológicos de la emoción, tal y como veremos. Así pues, pese a que cada teoría, tanto si pertenece a la corriente no cognitiva como a la cognitiva, es mucho más elaborada de lo que he presentado aquí, baste este breve resumen para situar la teoría de Nussbaum y el desarrollo que a continuación haremos del concepto de evaluación.

Por otra parte, merece la pena llamar la atención sobre el hecho de que la distinción entre teorías cognitivas y no cognitivas de la emoción no debe llevarnos a creer que existe una distinción entre emociones y razón. En la noción de evaluación se ve reflejada la sofisticación con la que los aspectos más animales e instintivos de nuestra racionalidad práctica y los aspectos más cognitivos o intelectuales se interrelacionan entre sí. Por ejemplo, la reacción de susto por la percepción de un peligro involucra una forma de miedo muy primitiva, mientras el miedo a la muerte es una forma de la emoción más cognitiva, puesto que implica reflexiones acerca de la finitud y el sufrimiento, pensamientos acerca del futuro, etc.

\section{LA NOCIÓN DE EVALUACIÓN EN PSICOLOGÍA}

La noción de evaluación (appraisal) es fundamental en las teorías de la emoción. Este concepto fue introducido en el ámbito de la psicología por Magda Arnold en 1960 en su libro Emotion and Personality. ${ }^{5}$ La evaluación es un proceso psicológico a través del cual el agente otorga un significado a un hecho o situación. Esta evaluación, a su vez, da lugar a una respuesta de

\footnotetext{
${ }^{5}$ Aunque en el presente artículo no podemos dedicar más espacio a esta cuestión, merece la pena señalar que, en el ámbito de la filosofía, existe toda una corriente de pensamiento, la teoría de los Valores, que aglutina a varios teóricos que presentan los valores como cualidades que tienen las cosas y que nosotros percibimos en ellas. Para Franz Brentano, por ejemplo, los valores se aprehenden intencionalmente (la consciencia se abre a -o se dirige hacia- ellos) y las emociones (o afectos, como él los denomina), también serían intencionales y estarían vinculadas al valor. Max Scheler, por su parte, habla de un percibir sentimental (como preferir, amar u odiar), y sostiene que la aprehensión de los valores se realiza a través del sentimiento. Ver Brentano (2002); Scheler (1941).
} 
atracción o aversión. Según este planteamiento, la emoción es una tendencia sentida hacia algo evaluado de forma intuitiva como bueno o beneficioso, o una tendencia sentida a rechazar algo intuitivamente evaluado como malo (ARNOLD, 1970). Ya en los ańos 80, las variables cognitivas y evaluativas se convierten en el foco de interés de los investigadores de la emoción. De esta época cabe destacar el trabajo de Nico Frijda (1986) y, por último, más recientemente, el de Klaus Scherer y sus colaboradores, que han realizado un enorme esfuerzo por elaborar modelos en los que las emociones son clasificadas según dieciocho o más dimensiones de evaluación (SCHERER, 2005; SCHERER; SCHORR; JOHNSTONE, 2001).

La evaluación sería una suerte de articulación entre un evento del mundo y el agente. La aparición de una emoción es un fenómeno dependiente del significado que un individuo haga de un hecho concreto. Nótese, por tanto, que la noción de evaluación se encuentra estrechamente ligada a la de interpretación, puesto que lo que estamos poniendo de relieve es que, mediante la evaluación, el agente interpreta un acontecimiento del mundo como bueno o malo, como potencialmente peligroso o potencialmente beneficioso, etc.

\section{RICHARD LAZARUS: UNA TEORÍA COGNITIVA-MOTIVACIONAL-RELACIONAL}

La teoría de las emociones de Richard Lazarus es uno de los referentes claros que Nussbaum tiene a la hora de elaborar su propia teoría. Como veremos, resulta fácil rastrear la influencia de las ideas de Lazarus en las concepciones de la autora. Lazarus (1991, p. 30-31) afirma:

Para generar una emoción, un encuentro adaptativo debe centrarse sobre un asunto personal, por así decirlo. Se trata de una transacción en curso entre la persona y el medio que tiene influencia sobre objetivos personales que se aportan al encuentro y respecto a los cuales las condiciones del medio son relevantes. ${ }^{6}$

Cada emoción se define en función del tipo particular de relación que una criatura establece entre ella misma y su entorno. Esto es lo que Lazarus denomina "temas relacionales nucleares" (core relational themes). Los temas relacionales se pueden definir, en consecuencia, como "[...] los perjuicios y beneficios personales presentes en cada relación persona-medio" (LAZARUS,

\footnotetext{
${ }^{6}$ Junto con Lazarus, otros de los psicólogos que han ejercido una importante influencia sobre
} Nussbaum son Oatley (1996), Ortony et. al. (1988) y Clore (1994). 
1991, p. 39). La evaluación sería el proceso a través del cual la persona construye el significado de las relaciones con su medio. Según Lazarus (1991, p. 39), el proceso de evaluación entraña un conjunto de elementos relacionados con la toma de decisiones que crean patrones de evaluación diferentes para cada una de las emociones. Es decir, que cada emoción individual se distingue por tener su propio patrón de componentes evaluativos.

El autor establece dos subgrupos para clasificar estas evaluaciones: las evaluaciones primarias y las evaluaciones secundarias. Las evaluaciones primarias determinan si alguna de las metas del agente está en juego o en peligro y las evaluaciones secundarias tienen que ver con cómo manejar una situación que afecta a las metas u objetivos del individuo de forma positiva o negativa (LAZARUS, 2000, p. 103). De un modo más específico, la evaluación primaria está formada por tres componentes: a) la evaluación de la relevancia del objetivo, esencial para que una transacción sea considerada de importancia para el bienestar personal; b) la evaluación de la congruencia o incongruencia del objetivo, que refiere a las dificultades o facilidades que pueda encontrar la persona para alcanzarlo (si las condiciones son favorables, se producirá una emoción de tono positivo, si son desfavorables, de tono negativo); y 3) el tipo de implicación del ego, que tiene que ver con el papel que desempeñan las diferentes metas a la hora de darle forma a la emoción, como, por ejemplo, los valores morales, los ideales del ego, el bienestar de las otras personas, o nuestros objetivos vitales (por ejemplo, la vergüenza, tiene que ver con un ideal del ego y con la imposibilidad de alcanzarlo debida a una falta propia).

Del mismo modo, las evaluaciones secundarias se componen de tres evaluaciones básicas que tienen que ver con: a) la evaluación de la culpabilidad y el crédito, esto es, tener un juicio sobre quién o qué es responsable del daño, beneficio, amenaza o lo que sea; b) la evaluación de las opciones de manejo, que surgen de la convicción personal de que podemos o no podemos actuar de forma satisfactoria para influir en el curso de un acontecimiento, ya sea para evitar un daño o para obtener un beneficio; y c) evaluación de las expectativas futuras, es decir, si esperamos que la relación de la persona con el entorno empeore o mejore (LAZARUS, 2000, p. 104-105).

En lo que respecta a los objetivos que un agente posee, éstos pueden venir dados por la biología, en el caso de las necesidades más básicas, pero también por un proceso de desarrollo personal en el que la sociedad posee un papel fundamental. La reacción emocional, según Lazarus, es el fenómeno más rico de la psicología, puesto que revela con gran profusión de detalles 
el modo en que nos relacionamos con las diferentes facetas de nuestra vida y con las particularidades de nuestro entorno material y social. La reacción emocional revela que un valor o un objetivo importante está sufriendo un perjuicio, corriendo un riesgo o progresando de la forma que esperamos.

Lazarus (1991, p. 6-7, citado por NUSSBAUM, 2008, p. 134) asegura:

A partir de una reacción emocional podemos aprender mucho de lo que a una persona le interesa en la relación con su medio o en la vida en general, cómo interpreta el mundo o a sí misma, y cómo se enfrenta a los perjuicios, peligros y retos. Ningún otro concepto en psicología revela con tanta riqueza el modo en que un individuo se relaciona con la vida y las especificidades de su medio físico y social.

Así pues, en resumidas cuentas, los procesos de evaluación y de manejo son producto de la personalidad más la interacción con el entorno. Por esta razón, Lazarus emplea la expresión "significado relacional" (relational meaning): este significado se basa en la importancia de lo que está ocurriendo alrededor de la persona y que tiene implicaciones para su bienestar. El rol de la evaluación, afirma el autor, consiste precisamente en integrar aquellas variables vinculadas a la personalidad, y aquellas variables que tienen que ver con el entorno, "[...] en un significado relacional basado en la relevancia de lo que está ocurriendo para el bienestar de la persona," (LAZARUS, 1991, p. 39). Más adelante veremos las similitudes que existen entre estas ideas y las postuladas por Nussbaum.

\section{LA RACIONALIDAD DE LAS EMOCIONES}

Tanto nosotros como otros organismos nos vemos obligados a estar atentos a lo que ocurre a nuestro alrededor: cada detalle puede ser un obstáculo, un peligro o una oportunidad para alcanzar la satisfacción de dichas necesidades o para el propio interés personal. Por esta razón, decimos que las emociones tienen una función adaptativa. El proceso de evaluación, no obstante, no sólo tiene que ver con aquellos medios, obstáculos, oportunidades, etc. que tengan que ver con una supervivencia desnuda, sino que también tienen que ver con otras facetas del ser humano relativas a su bienestar, a la imagen que tiene de sí mismo, y a sus relaciones con los demás. 
Nussbaum sostiene que las emociones son profundamente racionales. Lazarus también defiende que las emociones son racionales, o, por decirlo con Nussbaum, indisociables de nuestra racionalidad práctica (NUSSBAUM, 2008, p. 135). Según Lazarus, la capacidad para proyectar un objetivo y comprender los medios que son precisos para alcanzarlo, así como la de evaluar si se ha logrado con éxito un propósito o se ha fracasado, tiene que ser necesariamente emocional (LAZARUS, 1991, p. 468).

Las emociones están dotadas de racionalidad en el sentido de que valoran, resaltan o nos avisan sobre aspectos de la realidad que tienen una importancia crucial para nuestra vida y nuestro bienestar. Es a esto a lo que nos referimos cuando decimos que las emociones resultan fundamentales para nuestra racionalidad práctica. Sin ellas nos faltaría una pieza de nuestro mecanismo de toma de decisiones, del engranaje mental que nos permite deliberar y sopesar diferentes cursos de acción. Son de sobras conocidos los casos de pacientes con lesiones cerebrales que, privados de un cerebro sano, han visto rota la conexión entre procesos emocionales y de valoración e interpretación de lo que ocurre en el mundo. ${ }^{7}$ Esta dificultad es particularmente acusada en contextos en los que se requiere la interacción con otras personas. Privados de la competencia emocional necesaria para interpretar una situación y sopesar las alternativas adecuadas para enfrentarse a ella, estos pacientes se convierten en "inútiles sociales" (OVEJERO, 2010, p. 24). Cabría añadir que dicha inutilidad se extiende a todos los dominios de la vida hasta hacer a estos agentes incapaces de llevar una vida satisfactoria.

"Las emociones" - afirma Lazarus (2000, p. 98) - “[...] son el producto de la razón porque se derivan del modo en que valoramos lo que está sucediendo en nuestras vidas". La reacción emocional, por tanto, no es ciega e irracional. Ni tampoco podemos decir de ella que se encuentre totalmente desligada de motivos y razones, sino que responde al modo en que evaluamos un acontecimiento. Es por ello, prosigue Lazarus, por lo que podemos hablar de "emociones cognitivas". Las emociones poseen una "lógica implacable" (LAZARUS, 2000, p. 98) a tenor de la relación existente entre el yo y el mundo y las creencias que el agente tiene a propósito del mismo. Aun si las premisas que sostiene el individuo sobre su propio yo y su relación con el mundo son falsas o erróneas, la lógica de la emoción sigue estando ahí: la

7 Uno de los autores que ha estudiado y divulgado ampliamente estas cuestiones es Damasio (2006; 2005; 2010). Ver asimismo Goldberg (2002), Cortina (2011). 
persona siente de forma $\mathrm{X}$ porque cree $\mathrm{Y}$, y porque cree que $\mathrm{Y}$ tiene unas repercusiones para su propia vida y para su esquema de objetivos y fines.

Por otra parte, Lazarus critica duramente los planteamientos reduccionistas según los cuales el ser humano no es más que una criatura que actúa en función de la maximización de su propio interés. En primer lugar, porque las personas no siempre tienen claros cuáles son sus intereses o cuál es su esquema de objetivos y fines; y porque, además, con frecuencia no somos capaces de expresar verbalmente o de explicitarnos a nosotros mismos todas estas premisas. Por otra parte, esta forma de definir la racionalidad presupone que el propio autointerés es la máxima que rige toda acción, como si no hubiera valores que merecieran la pena más allá del propio autointerés. Cuestiones tales como el sacrificio en beneficio de los seres queridos, la cooperación con los amigos o los compañeros de trabajo, la manifestación de lealtad hacia algunas personas, la compasión para con el otro sufriente o la preocupación por la justicia son valores que, en ocasiones, también rigen nuestra acción, y que, como bien apunta Lazarus "[...] deberían constituirse como hitos de la civilización". Lazarus, por tanto, deja claro que ligar las emociones a la racionalidad no significa relacionarlas con el egoísmo. Así pues, tanto la avaricia como la compasión pueden ser consideradas racionales. Pero no por ello pensamos que las dos sean igual de buenas o encomiables.

En tercer lugar, Lazarus indica que, en ocasiones, actuamos de forma contraproducente para nuestros propios intereses, como cuando no juzgamos de forma adecuada un peligro (por exceso o por defecto), o cuando nos airamos en exceso por un suceso sin importancia, ocasionándonos a nosotros mismos gran malestar y dificultando nuestras relaciones con los otros. Comportarnos de tal modo puede parecer absurdo, o incluso estúpido, pero no debe considerarse, según Lazarus, irracional. Actuamos de este modo "[...] porque hemos valorado determinados sucesos de un modo particular, la mayoría de las veces basándonos en presunciones, motivos o creencias incorrectas." (LAZARUS, 2000, p. 99). Dichas creencias acaban desembocando, con frecuencia, en reacciones emocionales desproporcionadas o inapropiadas. Pero no son irracionales, puesto que ellas siguen su lógica implacable, en función de lo que creemos, lo que deseamos, y cómo pensamos conseguir lo que queremos.

Otra idea interesante que aporta Lazarus es que, a menudo, existen una serie de juicios erróneos que otorgan un cariz "absurdo" o "irracional" a nuestras emociones. Según Lazarus, lo que causa que realicemos juicios de forma errónea $y$, en consecuencia, manejemos forma inapropiada nuestras reacciones 
emocionales, son varias cosas. Destacan, por ejemplo, los casos en los que existe una condición patológica que impide a la persona realizar un razonamiento de forma adecuada (senilidad, retraso mental, psicopatía, daños cerebrales, etc.), o los casos en los que se da una falta de conocimiento, o, en otras palabras, se ignoran los detalles de las circunstancias y de lo que resulta apropiado para las mismas. Otra de las causas que merece la pena mencionar es la ambigüedad o incertidumbre a la hora de interpretar de manera adecuada lo que ocurre a nuestro alrededor: lo que parece un acto benévolo puede resultar un dulce envenenado, y lo que nos parece maldad puede no ser más que ineptitud.

En cualquier caso, Lazarus pone de relieve que existe una relación indisoluble entre creencias y emociones. ${ }^{8}$ Como decíamos al principio, para cada emoción, existiría lo que Lazarus denomina un tema relacional nuclear (core relational theme). Los temas relacionales de cada emoción podrían ser interpretados como la clase de cogniciones básicas relacionadas con cada emoción dirigida hacia un objeto intencional y que determina el tipo de actitud que se tiene hacia dicho objeto. Reproducimos a continuación la tabla en la que Lazarus expresa el tema relacional nuclear de cada emoción: (LAZARUS, 2000, p. 107) ${ }^{9}$

\begin{tabular}{|l|l|}
\hline \multicolumn{1}{|c|}{ Emoción } & \multicolumn{1}{c|}{ Tema relacional nuclear } \\
\hline Ira & Una ofensa degradante contra mí y los míos. \\
\hline Ansiedad & Enfrentarse a una amenaza incierta, existencial. \\
\hline Temor & Un peligro físico inmediato, concreto y sobrecargante. \\
\hline Culpa & Haber transgredido un imperativo moral. \\
\hline Vergüenza & No haberse mantenido a la altura del ideal del ego. \\
\hline Tristeza & Haber experimentado una pérdida irrevocable. \\
\hline Envidia & Esperar algo que tiene alguna otra persona. \\
\hline Celos & $\begin{array}{l}\text { Resentir a una tercera parte por la pérdida o amenaza del afecto o favor de } \\
\text { otra persona. }\end{array}$ \\
\hline Asco & Tomar o estar cerca de un objeto o idea indigesta (metafóricamente hablando). \\
\hline Felicidad & Hacer un progreso razonable en dirección a la realización del objetivo. \\
\hline Orgullo & $\begin{array}{l}\text { Fomento de la propia identidad del ego dando crédito a un objeto o logro } \\
\text { valorado, bien propio o de alguien con quien nos identificamos. }\end{array}$ \\
\hline Alivio & $\begin{array}{l}\text { Una condición molesta e incongruente para el objetivo que ha mejorado o se } \\
\text { ha eliminado. }\end{array}$ \\
\hline Esperanza & Temer lo peor pero anhelar algo mejor. \\
\hline
\end{tabular}

${ }^{8}$ Lazarus apunta que debemos a Aristóteles estas ideas. Ver Lazarus, 1991, p. 14.

${ }^{9}$ Es una reproducción dela tabla 3.4 que puede encontrarse en LAZARUS, 1991, p. 122. 


\begin{tabular}{|l|l|}
\hline Amor & $\begin{array}{l}\text { Desear o participar en el afecto, habitualmente aunque no necesariamente } \\
\text { recíproco. }\end{array}$ \\
\hline Gratitud & Aprecio por una donación altruista que aporta un beneficio personal. \\
\hline Compasión & Sentirse conmovido por el sufrimiento ajeno y desear ofrecer ayuda. \\
\hline $\begin{array}{l}\text { Experiencias } \\
\text { estéticas }\end{array}$ & $\begin{array}{l}\text { Emociones provocadas por estas experiencias pueden ser cualquiera de las } \\
\text { anteriores; no hay una secuencia especíica. }\end{array}$ \\
\hline
\end{tabular}

En resumen, un tema relacional nuclear se define por el significado que lleva aparejado a cada emoción. Así, uno no puede sentir gratitud por una ofensa u orgullo por no haber sido capaz de estar a la altura de un ideal del ego. Cada emoción responde a una lógica determinada, y en este sentido, sostiene Lazarus, las emociones están dotadas de racionalidad.

\section{LA NOCIÓN DE EVALUACIÓN EUDAIMONISTA: UN CONCEPTO CLAVE EN LA TEORÍA DE LAS EMOCIONES DE NUSSBAUM}

A continuación nos disponemos a examinar con más detenimiento la noción de evaluación eudaimonista, que como veremos, hace de la teoría de las emociones de Nussbaum una propuesta muy completa, puesto que dicho concepto vincula la cognición a un entramado de percepciones, evaluaciones, e ideas complejas acerca de lo que es bueno y conveniente para el agente.

Nussbaum recurre a las ideas de valor y evaluación para poner de manifiesto que las emociones no son fuerzas ciegas e irracionales. En las percepciones intencionales y las creencias relativas a la emoción, aduce, se contempla al objeto "[...] como investido de valor o importancia." (NUSSBAUM, 2008, p. 52). La propia autora recurre a los planteamientos de Richard Lazarus, cuya propuesta hemos examinado en el apartado anterior. Es fácil ver las similitudes que existen entre una teoría y otra, pero una delas diferencias más notables entre uno y otro es que Lazarus recurre al léxico de la psicología y expone su teoría remitiéndose a la función adaptativa que cumplen las emociones. Nussbaum, por su parte, emplea un aparato conceptual propio de la filosofía (como ocurre con la noción de eudaimonía). Si hubiera que resaltar un aspecto positivo de cada una de las teorías, podríamos decir que Lazarus resulta más claro y sistemático en su exposición, pero que Nussbaum añade cierta riqueza a su planteamiento al incluir ideas provenientes de la tradición filosófica y una reflexión sobre las implicaciones normativas de los marcos teóricos cognitivo y no cognitivo. Un ejemplo de esto último es que 
la autora defiende que, si las emociones son consideradas meros impulsos, la única forma de ejercer control sobre ellas será suprimiéndolas o subyugándolas, mientras que si concebimos las emociones como un entramado de cogniciones y evaluaciones, podremos influir en la forma que éstas toman. Esto, a su vez, tiene consecuencias a nivel práctico: no es lo mismo decir que somos agresivos contra las personas que no forman parte de nuestro entorno cercano de forma natural, y colegir que, en consecuencia, poco podemos hacer para cambiar estas tendencias, que defender que este tipo de inclinaciones se pueden educar desde la infancia mediante la reflexión.

Nussbaum reconoce que el concepto de valor resulta complicado de definir, pero da por buena la idea de que los juicios de emoción son una subclase de los juicios de valor. ${ }^{10}$ Esta afirmación implica, por tanto, que hay juicios de valor que no son juicios de emoción). Los juicios de emoción se refieren a objetos que poseen un papel importante en el esquema de objetivos y proyectos vitales de la persona. Para ejemplificar la distinción entre juicios de valor y juicios de emoción, la autora recurre al siguiente razonamiento: uno puede pensar que la actividad intelectual -en sentido genérico- es un bien. En consecuencia, es probable que crea que tanto las matemáticas como la filosofía son valiosas -puesto que son formas particulares de actividad intelectual-; sin embargo, el que se dedica a la filosofía considerará que ésta es una parte muy importante de su vida, y experimentará emociones en relación a esta actividad. No obstante, es posible que no experimente estas sensaciones con las matemáticas, porque, por mucho que piense que dedicarse a estudiarlas constituye una actividad valiosa en general, no tiene por qué sentirse particularmente concernido por ellas. Algunos de estos objetos, además, no pueden ser controlados totalmente por el agente que siente la emoción.

Nussbaum presenta aquí una idea fundamental en su planteamiento. El valor que se percibe en el objeto tiene una peculiaridad, a saber, que "[...] hace referencia al propio florecimiento de la persona". "El objeto de la emoción es visto como importante para algún papel que desempeña en la propia vida de la persona." (NUSSBAUM, 2008, p. 53). De este modo, no sentimos miedo por cualquier catástrofe que acontezca en el mundo, sino que sólo nos inspiran tal emoción las desgracias que tememos que lastimen aquello que amamos o

\footnotetext{
${ }^{10}$ Aun así, Nussbaum da una definición a grandes rasgos: "Podríamos mencionar las nociones de lo que merece ser procurado, lo que supone un buen empleo del tiempo o aquello de lo que parece bueno ocuparse." (NUSSBAUM, 2008, 53, nota 21). Una cuestión pendiente, a la que por razones de espacio no podemos atender, sería aclarar la relación entre el los valores culturales y el agente que valora y realiza juicios emocionales.
} 
que destruyan nuestros proyectos y aspiraciones. Igualmente, podemos sentir cierta desazón por la muerte de un ser humano cualquiera; consternación por la desaparición de alguien a quien hemos conocido; pero sólo verdadera desconsuelo y dolor cuando ese alguien era una persona querida que ha tenido un papel relevante en nuestra vida. "Las emociones" - dice Nussbaum (2008, p. 132) - "[...] son formas intensas de atención y de compromiso en las que el mundo es evaluado en su relación con el yo".

Nussbaum advierte, no obstante, que el hecho de que desde el punto de vista emocional concibamos los objetos del mundo de este modo, no implica que éstos sean vistos como meros instrumentos o herramientas para la satisfacción del agente (NUSSBAUM, 2008, p. 53). ${ }^{11}$ Creer que una persona en particular está investida de una enorme importancia para mí, no excluye que también sea capaz de considerar que dicha persona posee una valía intrínseca y es un fin en sí misma. Nuestra autora señala que no hay egoísmo aquí, sino, simplemente, auto-referencia. ${ }^{12} \mathrm{Si}$ mis emociones se focalizan en un ser querido, y no en otro ser humano, es porque éste es una parte central de $m i$ vida. En este sentido, señala Nussbaum (2008, p. 53),

Las emociones poseen un carácter local: adoptan un lugar peculiar dentro de mi propia vida [...]. Incluso cuando se interesan por acontecimientos que tienen lugar a distancia o eventos del pasado, es porque la persona ha conseguido investir tales sucesos de cierta importancia dentro de su propio esquema de fines y objetivos. (grifo nosso).

Otra manera de expresar la idea de que desde la perspectiva de las emociones otorgamos un valor a los objetos en función de la importancia que ocupan en nuestro esquema de objetivos y fines es decir que las emociones tienen un carácter eudaimonista ${ }^{13}$. Esto quiere decir que se encuentran estrechamente

\footnotetext{
${ }^{11}$ Nussbaum cuestiona aquí la postura de Robert Solomon, quien sostiene que el objetivo de las emociones consiste en "maximizar" la "dignidad personal y la autoestima" (1993, p. 160). Esto, a juicio de Nussbaum, las torna excesivamente egoístas. La autora, por el contrario, defiende que las emociones poseen un componente eudaimonista, pero que aun así pueden "[...] incluir el bienestar de otros seres lejanos como un elemento de valor en mi esquema de fines y objetivos". Este sería el caso de la compasión, experiencia emocional por la que nos dolemos del sufrimiento del otro (NUSSBAUM, 2008, p. 53, nota 22).

${ }^{12}$ La autora insiste mucho en esta idea en una discusión crítica con Ben-ze'ev recogida en Nussbaum (2004).

${ }^{13} \mathrm{La}$ autora sostiene que prefiere emplear el término "eudaimonista" (eudaimonistic en el original) en vez de "eudemónico" (eudaemonistic en el original) porque considera que este último se ha asociado con frecuencia a una perspectiva particular, de corte utilitarista, según la cual la felicidad entendida
} 
relacionadas con el florecimiento del agente que las experimenta, es decir, con aquellas cosas que son buenas para él (NUSSBAUM, 2008, p.54). En consecuencia, una teoría ética eudaimonista trata de dar respuesta a la pregunta acerca de cómo ha de vivir el ser humano, de qué es bueno para nosotros. Esto es precisamente a lo que nos referimos cuando traemos a colación la idea de florecimiento humano. El florecimiento refiere a una vida humana plena, y la vida humana plena es aquella en la que no faltan aquellas cosas a las que atribuimos un valor. Los seres humanos no buscamos únicamente sobrevivir, sino que queremos hacerlo con cierta calidad. ${ }^{14}$

De nuevo, Nussbaum (2008, p. 54) incide aquí en que "[...] en una teoría eudaimonista, las acciones, relaciones y personas incluidas en ella no son valoradas simplemente debido a la relación instrumental que puedan guardar con la satisfacción del agente". La autora insiste en que este es un error habitual cuando se habla de teorías eudaimonistas, puesto que éstas son entendidas de forma reduccionista desde el prisma del utilitarismo, a causa de una traducción inexacta de "felicidad" por eudaimonía. ${ }^{15}$ Esta es una concepción muy estrecha de lo que podemos entender por felicidad, esencialmente ligada, como ya hemos indicado, a la propia satisfacción y al placer. En las relaciones personales, sean éstas de tipo cívico, amistoso o amoroso, existe un deseo de beneficiar al otro por sí mismo, y ello puede constituir una parte esencial de la eudaimonía propia (NUSSBAUM, 2008, p. 55). De este modo, un individuo puede perseguir la justicia social como fin en sí mismo, aun si de ello no se deriva una ventaja inmediata para él. Asimismo, un individuo que posea esta creencia, también se sentirá emocionalmente vinculada a la misma. Si la justicia social forma parte de mi idea de eudaimonía, me sentiré afligida cuando ésta no llegue a hacerse efectiva y, del mismo modo, sentiré alegría cuando considere que sí lo hace, puesto que algo que yo reconozco como un objetivo vital, como un elemento fundamental de mi propio florecimiento, habrá sido logrado.

como placer constituye el bien supremo. Nussbaum prefiere referirse explícitamente a la noción de eudaimonía tal como era entendida en la antigua Grecia, de tal modo que la palabra toma un sentido más abarcador y es compatible con diversas teorías del bien. (NUSSBAUM, 2008, p. 54, nota 23).

${ }^{14}$ Una obra reciente que puede servir al lector para familiarizarse con el enfoque de las capacidades y la idea de florecimiento humano es Nussbaum (2012).

${ }^{15} \mathrm{La}$ autora señala que este es un error de interpretación y, haciendo alusión a las argumentaciones de J.L. Austin ( 1970, 1-31), aboga por una corrección del mismo. "El término "felicidad» resulta engañoso si se interpreta que el fin u objetivo es un estado de placer o satisfacción. Como muestra Austin, la palabra inglesa (happiness) tenía un alcance mayor al incluir las acciones buenas que no producen placer." (NUSSBAUM, 2008, p. 54, nota 25). 
El hecho de reconocer la importancia que tiene por sí mismo un objeto del mundo no excluye que el agente pueda ver ese mismo objeto como parte de su esquema de fines. Por este motivo, en los casos en los que acontecen emociones negativas, el yo se siente destrozado. Dichas emociones "[...] tienen que ver conmigo mismo y con lo mío, con mis planes y objetivos, con lo que es importante en mi propia concepción (o impresión embrionaria) de lo que significa vivir bien.” (NUSSBAUM, 2008, p. 55). En conclusión, desde la experiencia emocional se percibe el mundo desde el punto de vista del agente, "[...] trasladando los acontecimientos a la noción de éste de lo que posee valor o importancia de carácter personal.” (NUSSBAUM, 2008, p. 55).

\section{LA TEORÍA ÉTICA Y LA REFERENCIA AL YO EN LA CONCEPCIÓN EVALUATIVA EUDAIMONISTA}

Como ya hemos señalado, uno de los aspectos más relevantes en concepción eudaimonista es la referencia al yo del esquema de objetivos y proyectos que se encuentra implícito en el punto de vista emocional. Desde la experiencia emocional, por tanto, no podemos sino contemplar el mundo desde una perspectiva íntima, y calibrar las cosas desde nuestra concepción de lo que importa y lo que merece ser querido. "En pocas palabras" - dirá Nussbaum (2008, p. 75) -, "[...] las valoraciones asociadas con las emociones son evaluaciones desde mi perspectiva, no desde un punto de vista imparcial; encierran una referencia ineliminable al yo". Pero ya hemos visto anteriormente que el hecho de que en las emociones esté presente el punto de vista valorativo y personal, no implica que éstas sean necesariamente egoístas. En la compasión, por ejemplo, nos dolemos del sufrimiento de otro; y en una concepción de la eudaimonía en la que la búsqueda de la justicia tiene un papel relevante, el bien de los demás es considerado como un bien para uno mismo.

Pese a todo, hay que tener en cuenta que esta caracterización de las emociones "[...] les da un carácter localizado, y en ese sentido las enfrenta a las formas de moralidad defensoras de una completa imparcialidad." (NUSSBAUM, 2008, p. 76). La experiencia emocional, por tanto, se encuentra siempre condicionada por esa necesaria referencia al yo, por los propios apegos, valores y planes vitales, y por ello es difícil que las emociones no se encuentren restringidas al círculo de las cosas cercanas que nos importan y nos influyen de manera directa. Esta es, precisamente, una de las críticas que se podrían esgrimir contra las propuestas éticas que se basan en la compasión: 
su parcialidad y su incapacidad para extenderse más allá del reducido círculo de nuestros allegados.

Por otra parte, cable plantear una pregunta importante que la propia autora formula: ¿son todas las emociones eudaimonistas?, “[... ] ¿hacen referencia todas ellas a mis objetivos y proyectos importantes?, ¿Encierran la totalidad de las mismas el elemento autorreferencial que constituye el núcleo de la estructura eudaimonista?" (NUSSBAUM, 2008, p. 76). La autora no dedica mucho espacio a solventar estas dudas, pero reconoce que, en ocasiones, una emoción puede no ser eudaimonista. Este sería el caso de asombro. Cuando experimentamos esta emoción el objeto ejerce una atracción sobre el agente. En el asombro la persona es consciente -y ello es lo que otorga fuerza a la emociónde que el objeto parece ser valioso, especial, excepcional; pero no es consciente, o sólo lo es en grado mínimo, del papel que tal objeto puede tener en relación a sus planes y objetivos vitales. Nussbaum finaliza esta reflexión indicando que, si bien el esquema eudaimonista puede aplicarse a todas las emociones en general, tampoco es preciso ser dogmáticos al respecto, ya que, en algunos casos, como en el asombro, no parece desempeñar un rol fundamental.

Algunas emociones nos producen unas reacciones mucho más intensas que otras. Nussbaum afirma que dichas diferencias de intensidad suponen una intencionalidad dirigida al objeto, y que ocurren en función de la importancia que se confiera al objeto y a lo que a éste le suceda. La pérdida de un objeto trivial, por ejemplo, no es motivo de aflicción para nosotros. Existe un umbral por debajo del cual ni siquiera experimentamos emociones. Por encima de ese umbral, sin embargo, "[...] las diferencias de intensidad son ocasionadas por las diferencias en la valoración eudaimonista” (NUSSBAUM, 2008, p. 78). En una emoción como la cólera, por ejemplo, la intensidad con que sentimos dicho afecto variará en función de la magnitud del daño que creamos que se ha infligido. En el caso de la aflicción, el alcance de la pérdida será lo que determine con qué grado de fuerza sentimos la afección.

La autora advierte que las emociones poseen cierta tendencia a ser desproporcionadas respecto de sus objetos, ya que las personas solemos otorgar una importancia excesiva a objetos que en realidad no la tienen. En ocasiones, sufrimos por pérdidas triviales o hacemos de un objeto particular una fuente de preocupaciones y angustias. Un ejemplo de esto sería una persona avara, que desea adquirir riquezas y conservarlas de forma casi patológica. La avaricia sería un afecto desordenado que provocaría un apego exagerado por el objeto de la emoción - el dinero, las riquezas -, de tal modo que éstos no serían ya 
medios para conseguir una vida buena, sino que se convertirían en un fin (MARINA; LÓPEZ-PENAS, 1999, p. 79). En este y otros ejemplos, como la veneración que se puede sentir por una estrella deportiva o la pena que experimentan algunas personas cuando pierde su equipo favorito, queda patente que en ocasiones los seres humanos tenemos dificultades a la hora de calibrar con sensatez el valor de las cosas. Sin embargo, aun en los casos descritos, en los que la evaluación no es acertada y sensata, "[...] es la naturaleza de la valoración eudaimonista la que explica la intensidad de la emoción” (NUSSBAUM, 2008, p. 79).

En cualquier caso, lo que nos interesa destacar aquí es que cuando realizamos juicios éticos desde las emociones, éstos tienden habitualmente a la parcialidad, debido al estrecho vínculo de las emociones con el yo y con lo que importa a ese yo. Pero ello no es óbice para que las emociones puedan ser consideradas una materia ética valiosa. Las emociones no son ni buenas ni malas en sí mismas, pero pueden ser estudiadas para descubrir las trampas que encierran y los sesgos que entrañan, y pueden ser, asimismo, un precioso recurso para la educación moral. Aunque esta es una cuestión que precisaría de un extenso desarrollo, no disponemos de espacio aquí para tratarla con el detenimiento que merece.

\section{LAS EMOCIONES Y NUESTRA DEPENDENCIA DE LOS BIENES EXTERNOS}

En opinión de Nussbaum, uno de los puntos que más interés tiene del pensamiento de los estoicos acerca de las emociones es que éste da cuenta de cómo desde el punto de vista de las emociones nos hacemos conscientes de nuestra vulnerabilidad ante el mundo. Según los antiguos estoicos, aquellos juicios que se identifican con las emociones son de una clase especial, porque todos tienen en común que conciernen a lo que Nussbaum denomina "bienes externos". Por bienes externos no debemos entender únicamente bienes de tipo material que se encuentran situados fuera del cuerpo de la persona, sino aquellos eventos del mundo -sean estos externos o internos- que tienen algún interés para el agente y que quedan fuera su capacidad de control (NUSSBAUM, 2008, p. 64). Es decir, que también aquellas cosas que ocurren dentro del cuerpo (por ejemplo, aquellas que tienen que ver con la salud y la enfermedad) pueden ser consideradas "bienes externos", porque existe una medida en la que no podemos controlarlas a voluntad. La tesis de Nussbaum es que las emociones nos "[...] vinculan a elementos que consideramos importantes para nuestro bienestar pero que no controlamos plenamente". Lo que se pone de relieve en la experiencia 
emocional es nuestra falta de control ante los eventos del mundo. Por este motivo hemos indicado que las emociones se encuentran estrechamente vinculadas a la conciencia de nuestra fragilidad (NUSSBAUM, 2008, p. 66).

Otro filósofo contemporáneo de la corriente cognitiva, Aaron BenZe'ev, sostiene una postura similar a Nussbaum en lo referente a la relación existente entre las emociones y nuestra condición de seres vulnerables. El autor sostiene que las reacciones afectivas se encuentran indisolublemente ligadas a nuestra "existencia contingente", ya que "[...] nuestra posible muerte se encuentra siempre en el trasfondo de nuestra existencia: nos recuerda nuestra propia vulnerabilidad" (BEN'ZE-EV, 2000, p. 16) ${ }^{16}$ Este mismo trasfondo de autoconciencia de la propia finitud también nos confronta con nuestra dependencia de elementos externos que no podemos controlar.

La cuestión de la capacidad de control sobre las emociones o de autocontrol es un punto clave en el que se hace patente la escisión entre las teorías éticas estoicas antiguas y la interpretación renovada de Nussbaum de estas teorías. Los estoicos postularon que las emociones debían ser extirpadas de la vida humana ya que, en la medida en que nos revelan como vulnerables ante acontecimientos que no controlamos, suponen una ruptura de nuestra autosuficiencia (NUSSBAUM, 2003).

La autora sostiene que la doctrina estoica entiende que nuestro pensamiento es de naturaleza totalmente controlable, y que la virtud es algo susceptible de ser alcanzado por nuestros propios medios. Pero la mayoría de nosotros, por el contrario, "[...] tendemos a pensar que ningún estado o actividad mental se halla completamente bajo nuestro control.” (NUSSBAUM, 2008, p. 65).

En el programa ético de los estoicos, explica Nussbaum, la concepción cognitiva-evaluadora de las emociones tenía como finalidad enseñar cómo las sociedades y los individuos que las componían podían librarse de algunas emociones dañinas, como por ejemplo la ira, la envidia o el temor. En efecto, los autores estoicos instan al lector a alcanzar un estado de imperturbabilidad desterrando este tipo de emociones, motivo por el que Nussbaum les acusa de mantener una postura excesiva respecto a las mismas, porque interpreta que para los estoicos todas las emociones son malas (NUSSBAUM, 1987). La propuesta de la autora consiste en reformular el punto de vista estoico,

\footnotetext{
${ }^{16} \mathrm{El}$ autor llega a afirmar, incluso, que algunos trastornos de tipo afectivo, como la ansiedad y la depresión, se encuentran ligados con estas cuestiones existenciales y con la conciencia de nuestra vulnerabilidad y control imperfecto.
} 
aceptando que existe un conjunto de emociones que resultan perniciosas desde el punto de vista social y moral. El asco, que suele proyectarse hacia grupos sociales que se perciben de forma hostil, constituye un buen ejemplo de este tipo de emociones peligrosas (NUSSBAUM, 2006, 2008).

\section{Conclusiones}

Dado que las teorías no cognitivas prestan especial atención a la función adaptativa de las emociones, podría parecer que la teoría de Nussbaum está peor pertrechada para dar cuenta de las mismas, por tratarse de una propuesta que se centra en las cogniciones vinculadas a las mismas. Sin embargo, remitiéndonos al concepto de evaluación eudaimonista, podemos ver que la teoría de Nussbaum no encuentra dificultades para dar cuenta de dicha función adaptativa, ni tampoco para dar respuesta al apremio y la fuerza de las emociones. La evaluación eudaimonista no es un mero juicio sobre el mundo, sino que involucra una evaluación de la situación o el objeto en cuestión que tiene en cuenta los propósitos y fines vitales del agente, sea éste un animal o un ser humano, y de cómo dichos propósitos pueden ser logrados o frustrados, resultando esta evaluación fundamental para el bienestar del individuo. La noción de evaluación eudaimonista, por tanto, tiene en cuenta que las emociones son un mecanismo adaptativo al servicio de la supervivencia y el bienestar del organismo, presentándolas como una suerte de gozne entre éste y el mundo externo. Pero la concepción evaluativa eudaimonista no sólo atiende a esta dimensión de las emociones, sino que además explica su relación íntima con el yo, así como su carácter urgente, y es capaz de abordar la complejidad de las mismas, puesto que tiene en cuenta cómo los pensamientos, las creencias, la imaginación y el aprendizaje social influyen constantemente en la forma que éstas toman.

GIL, Marta. The notion of eudaimonistic evaluation in Martha Nussbaum's cognitive theory of emotions. Trans/form/ação, Marília, v. 39, n. 3, p.191-210, Jul./Set., 2016.

\footnotetext{
ABSTRACT: This article presents eudaimonistic evaluation as an articulation between the agent and the objects of the world. We will argue that, thanks to this notion, Nussbaum's theory of emotions is appropriate to accounting for the adaptive function of emotions. This notion is useful for shedding light on the cognitive aspects of emotion. We will see how Nussbaum's ideas are based on evaluative theories from psychology, and we will explain in detail her proposals. In our explanation, we will
} 
deal with other questions such as the rationality of emotions, the relationship between emotions and human flourishing, and the link between emotions and our vulnerable nature.

KEYWORDS: emotions, Martha Nussbaum, cognitive theories, evaluation, eudaimonism, rationality

\section{REFERENCIAS}

ARNOLD, Magda B. Emoción y personalidad. Buenos Aires: Losada, 1970.

AUSTIN, J. L. Agathon and eudaimonia in the ethics of Aristotle. In: . Philosophical papers. Nueva York: Oxford University Press, 1970. p. 1-13.

BEN-ZE'EV, A. The subtlety of emotions. Cambridge, Massachusetts: MIT Press, 2000.

BRENTANO, F. Psicología. Madrid: Revista de Occidente, 1935.

CLORE, G. L. Why emotions require cognition. In: EKMAN, P.; DAVIDSON, R. J. (Ed.). The nature of emotion: fundamental questions. Oxford: Oxford University Press, 1994. p. 181-191.

CORTINA, A. Neuroética y neuropolitica: sugerencias para la educación moral. Madrid: Tecnos, 2011.

DAMASIO, A. R. En busca de Spinoza: neurobiología de la emoción y los sentimientos. Barcelona: Crítica, 2005.

. El error de Descartes: la emoción, la razón y el cerebro humano. Barcelona: Crítica, 2006.

. Yel cerebro creó al hombre: ¿cómo pudo el cerebro generar emociones, sentimientos, ideas y el yo? Barcelona: Destino, 2010.

DELANCEY, C. Passionate engines. Oxford; New York: Oxford University Press, 2002.

EKMAN, P. Basic emotions. In: DALGLEISH, T.; POWER, T. (Ed.). The handbook of cognition and emotion. New York: John Wiley \& Sons, 1999. p. 45-70.

Biological and cultural contribution to body and facial movement in the expression of the emotions. In: RORTY, A. (Ed.). Explaining emotions. Berkeley; Los Angeles: London: University of California Press, 1980. p. 73-102

FRIJDA, N. H. The emotions. Cambridge: Cambridge University, 1986.

GOLDBERG, E. El cerebro ejecutivo: lóbulos frontales y mente civilizada. Barcelona: Crítica, 2002.

GOLDIE, P. The emotions: a philophical exploration. Oxford: Oxford University Press, 2002. GRIFFITHS, P. E. What emotions really are. Chicago: University of Chicago Press, 1997.

LAZARUS, R. S. Emotion and adaptation. New York: Oxford University Press, 1991. Estrés y emoción. Bilbao: Desclée de Brouwer, 2000. (Biblioteca De Psicología. V. 106). 
GIL, M.

MARINA, J. A.; LÓPEZ PENAS, M. Diccionario de los sentimientos. Barcelona: Anagrama, 1999.

NUSSBAUM, M. The stoics and the extirpation of the passions. Apeiron, v. 20, n. 2, p. 129-178, 1987.

. La terapia del deseo: teoría y práctica en la ética helenística. Barcelona: Paidós, 2003.

2004. . Responses. Philosophy and Phenomenological Research, v. LXVIII, n. 2, p. 473-486, . El ocultamiento de lo humano: repugnancia, vergüenza y ley. Buenos Aires: Katz, 2006. . Paisajes del pensamiento: la inteligencia de las emociones. Barcelona: Paidós, 2008.

. Crear capacidades: propuesta para el desarrollo humano. Barcelona: Paidós, 2012. OATLEY, K.; JENKINS, J. M. Understanding emotions. Oxford: Blackwell, 1996.

ORTONY, A.; CLORE, G. L.; COLLINS, A. The cognitive structure of emotions. Cambridge: Cambridge University Press, 1988.

OVEJERO, F. Emociones razonables. Claves De Razón Práctica, n. 203, p. 22-28, 2010.

PLUTCHIK, R. Emotion: a psychoevolutionary synthesis. Nueva York: Harper and Row, 1980.

PRINZ, J. Emotion, psychosemantics and embodied appraisals. In: HATZIMOYSIS, A. Philosophy and the emotions. Cambridge: Cambridge University Press, 2003.

. Which emotions are basic? In: EVANS, D.; CRUSE, P. (Ed.). Emotion, evolution and rationality. Oxford: Oxford University Press, 2004a. Cap. 4.

. Gut reactions: philosophy of mind series. Oxford; New York: Oxford University Press, 2004b.

. Emotion: competing theories and philosophical issues. In THAGARD, P. (Ed.). Philosophy of psichology and cognitive science. Holanda: Elsevier, 2007. p. 247-266.

SCHELER, M. Ética: nuevo ensayo de fundamentación de un personalismo ético. Madrid: Revista de Occidente, 1941.

SCHERER, K. R. What are emotions? And how can they be measured? Social Science Information Sur Les Sciences Sociales, v. 44, n. 4, p. 695-729, 2005.

. SCHORR, A.; JOHNSTONE, T. (Ed.). Appraisal processes in emotion. Oxford; Nueva York: Oxford University Press, 2001.

SOLOMON, R. C. The passions. Indianapolis: Hackett Publishing Company, 1993.

ZAJONC, R. B. Feeling and thinking: preferences need no inferences. American Psychologist, v. 35, n. 2, p.151-175, 1980.

. On the primacy of affect. American Psychologist, v. 39, n. 2, p. 117-123, 1984.

Recebido em 17/12/2015

Aceito em 20/04/2016 